\title{
Introduction to Social Media and e-Business Transformation Minitrack
}

\author{
Christy M.K. Cheung \\ Department of Finance and \\ Decision Sciences \\ Hong Kong Baptist University \\ ccheung@hkbu.edu.hk
}

\author{
Matthew K.O. Lee \\ Department of Information \\ Systems \\ City University of Hong Kong \\ ismatlee@cityu.edu.hk
}

\author{
Christian Wagner \\ School of Creative Media \\ City University of Hong Kong \\ c.wagner@cityu.edu.hk
}

Social media are online platforms that facilitate global collaboration and sharing amongst users. New social media applications in e-business and e-commerce appear on a daily basis and result in enormous shocks to the ecosystem of individuals and businesses. This minitrack provides a forum for the exchange of research ideas and best practices related to social media in e-business environments. It also aims to raise awareness in terms of the latest developments in social media, and address the challenges of using social media. This year, eight papers were selected for inclusion in the proceedings.

The first paper, "Perceived Individual Risk of Co-innovation in Collaborative Innovation Networks" by Kaveh Abhari, Elizabeth Davidson, Bo Xiao develops and empirically tests a model to measure the actors' perception of risk. The authors identify the model structure and empirically test the nomological network, demonstrating the relationship between the perceived individual risk and co-innovation behavior. The study contributes to our understanding of how to cultivate collective innovation while preserving the interest of the individual contributors.

The next paper by Jisun An, Haewoon Kwak, Bernard J. Jansen, "Automatic Generation of Personas Using YouTube Social Media Data", shows how the automatic persona generation system pulls social media interactions from social media platforms to develop personas in real time. The paper also demonstrates that how actual user data reduces time and cost for generating personas relative to traditional methods.

Drawing from prior research on online conformity, Yanli Jia and Libo Liu investigated how different types of social information influence users' purchase decision in their paper "Who do We Listen to More: Opinion Leaders or Friends? The Social Function of Conformity Behavior in Social Commerce." The paper has a specific focus on the moderating role of gender in the research model. The data of this study was crawled from a social commerce site in Asia.
The fourth paper, "How Questions and Answers Shape Online Marketplaces: The Case of Amazon Answer" by Warut Khern-am-nuai, Hossein Ghasemkhani, and Karthik Kannan, uses the difference-in-differences analysis to examine the effect of Q\&A elements on product sales. The authors collected data from two online shopping platforms. The results offer managers some guidelines to develop policies to gather necessary answers to questions asked on the Q\&A platform.

"The Roles of Transactive Memory Capability in a Social Media Environment", by Hyo Jun Kim, Kee-Young Kwahk defines the concept of transactive memory capability and examines its roles in the social media environment. Four determinants, social interaction, social presence, self-monitoring, and core self-evaluation are identified, and transactive memory capability has an impact on job performance in the social media environment.

Drawing on the diversity literature, Lili LIU, Ayoung Suh, Christian Wagner developed and empirically tested a research model explaining funder's intention to donate money in charitable crowdfunding projects. Empathy and perceived credibility of charitable crowdfunding were identified as two important determinants of intention to donate. Website quality and project content quality positively influence empathy and perceived credibility. This paper contributes to our understanding of individuals' donation behavior in online micro charities.

The next paper, 'How to Increase Users' Social Commerce Engagement? A Technology Attractiveness Model" by Xiao-Liang Shen, Yang-Jun Li, Yongqiang Sun, Zhenjiao Chen, Kem Z.K. Zhang, Sesia J. Zhao, uses attractiveness theory to develop a research model explaining how technology attractiveness promotes user involvement, which in turn affects social commerce engagement. The model was empirically with data collected from a social commerce site from China.

The final paper, "An Exploratory Study on Consumers' Attention towards Social Media 
Advertising: An Electroencephalography Approach? A Technology Attractiveness Model" by Huichih Wang and, Hersen Doong, demonstrates consumers' attention towards social media advertising using an electroencephalographic (EEG) approach. The authors also focused on the gender differences in sensing humor in advertisement displayed on social media. The results of this study give insights for understanding social media advertising.

We thank the authors for submitting their work to make this another engaging minitrack. We hope you enjoy the papers and their presentation at the conference. 